\title{
Some inequalities involving two generalized beta functions in $n$ variables
}

\author{
Mustapha Raïssouli ${ }^{1,2^{*}}$ (D) and Salma I. El-Soubhy ${ }^{3}$
}

"Correspondence:

raissouli.mustapha@gmail.com

'Department of Mathematics,

Science Faculty, Qassim University,

Qassim, Saudi Arabia

2Department of Mathematics,

Science Faculty, Moulay Ismail

University, Meknes, Morocco

Full list of author information is

available at the end of the article

\section{Springer}

\begin{abstract}
The beta and gamma functions have recently seen several developments and various extensions because of their nice properties and interesting applications. The contribution of this paper falls within this framework. After introducing a generalized gamma function and two generalized beta functions in several variables, we investigate some inequalities involving these generalized functions.
\end{abstract}

MSC: 26D15;26D99

Keywords: Beta function; Gamma function; Beta function in $n$ variables; Extended beta function in $n$ variables; Generalized beta function in $n$ variables; Inequalities

\section{Introduction}

The standard beta and gamma functions are respectively defined by

$$
\begin{aligned}
& \forall x, y>0, \quad B(x, y)=: \int_{0}^{1} t^{x-1}(1-t)^{y-1} d t, \\
& \forall x>0, \quad \Gamma(x)=: \int_{0}^{+\infty} t^{x-1} e^{-t} d t .
\end{aligned}
$$

Such functions play an important role in mathematical analysis and have wide applications in various contexts of mathematics and physics. An interesting relationship expressing a connection between $B$ and $\Gamma$ is given by

$$
\forall x, y>0, \quad B(x, y)=\frac{\Gamma(x) \Gamma(y)}{\Gamma(x+y)} .
$$

Otherwise, it is well-known that $\Gamma(x+1)=x \Gamma(x)$ for all $x>0$. For further properties and applications of the beta and gamma functions, we refer the interested reader to [2-6, 917, 23], for instance.

The previously defined functions $B$ and $\Gamma$ have been extended in the literature, see [7, $8,19]$. In [7], Chaudhry et al. introduced the following extended beta function:

$$
\forall x, y>0, \quad B(x, y ; a)=: \int_{0}^{1} t^{x-1}(1-t)^{y-1} e^{-a / t(1-t)} d t
$$

(c) The Author(s) 2021. This article is licensed under a Creative Commons Attribution 4.0 International License, which permits use sharing, adaptation, distribution and reproduction in any medium or format, as long as you give appropriate credit to the original author(s) and the source, provide a link to the Creative Commons licence, and indicate if changes were made. The images or other third party material in this article are included in the article's Creative Commons licence, unless indicated otherwise in a credit line to the material. If material is not included in the article's Creative Commons licence and your intended use is not permitted by statutory regulation or exceeds the permitted use, you will need to obtain permission directly from the copyright holder. To view a copy of this licence, visit http://creativecommons.org/licenses/by/4.0/. 
and the extended gamma function

$$
\forall x>0, \quad \Gamma_{a}(x)=: \int_{0}^{\infty} t^{x-1} e^{-t} e^{-a / t} d t,
$$

where $a \geq 0$ is a real parameter.

Another extension of $B(x, y)$ has been introduced by Choi et al. in [8] and reads as follows:

$$
\forall x, y>0, \quad B(x, y ; a, b)=: \int_{0}^{1} t^{x-1} e^{-a / t}(1-t)^{y-1} e^{-b /(1-t)} d t,
$$

where $a, b \geq 0$. It is clear that $B(x, y ; 0)=B(x, y), \Gamma_{0}(x)=\Gamma(x)$ and $B(x, y ; a, a)=B(x, y ; a)$, for any $x, y>0$ and $a \geq 0$. For more properties about $B(x, y ; a)$ and $\Gamma_{a}(x)$, see $[7,19]$, and for those of $B(x, y ; a, b)$ we can consult [8].

Further extensions of the beta and gamma functions have been investigated in the literature. We recall that the notation $(\lambda)_{m}$, when $m$ is a nonnegative integer and $\lambda \in \mathbb{R}$ is nonzero, stands for the Pochhammer symbol defined by [20]

$$
(\lambda)_{m}=\lambda(\lambda+1) \cdots(\lambda+m-1), \quad \text { with }(\lambda)_{0}=1 .
$$

In [19], Özergin et al. introduced the generalized beta and gamma functions defined respectively by

$$
\begin{aligned}
& \forall x, y>0, \quad B_{p}^{(c, d)}(x, y)=: \int_{0}^{1} t^{x-1}(1-t)^{y-1}{ }_{1} F_{1}\left(c ; d ; \frac{-p}{t(1-t)}\right) d t, \\
& \forall x>0, \quad \Gamma_{p}^{(c, d)}(x)=: \int_{0}^{\infty} t^{x-1}{ }_{1} F_{1}\left(c ; d ;-t-\frac{p}{t}\right) d t,
\end{aligned}
$$

where $c, d>0$ and $p \geq 0$. Here, the notation ${ }_{1} F_{1}(a ; b ; z)$ refers to the confluent hypergeometric function (CHF) defined through [18]

$$
{ }_{1} F_{1}(a ; b ; z)=: \sum_{m=0}^{\infty} \frac{(a)_{m}}{(b)_{m}} \frac{z^{m}}{m !} .
$$

If $a, b$ are integers, $a<0$, and either $b>0$ or $b<a$, then the series (1.7) converges and ${ }_{1} F_{1}(a ; b ; z)$ is a polynomial function. If $b$ is an integer with $b \leq 0$ then the series (1.7) diverges and ${ }_{1} F_{1}(a ; b ; z)$ is undefined. Note that ${ }_{1} F_{1}(a ; b ; 0)=1$. If $a, b-a>0$ then we have [19]

$$
{ }_{1} F_{1}(a ; b ; z)=\frac{\Gamma(b)}{\Gamma(a) \Gamma(b-a)} \int_{0}^{1} u^{a-1}(1-u)^{b-a-1} e^{z u} d u .
$$

Making the substitution $t=1-u$ in the latter integral formula, it is easy to check that

$$
{ }_{1} F_{1}(a ; b ; z)=e^{z}{ }_{1} F_{1}(b-a ; b ;-z) .
$$

The (CHF) stems its importance in the fact that it contributes as a good tool for solving many mathematical problems. It also appears as a solution of some partial differential equations, playing an important role in various mathematical areas. See $[6,18]$, for instance. 
Remark 1.1 The real functions $\Gamma(x)$ and $B(x, y)$, as well as their previous extensions, are also well-defined when the variables $x$ and $y$ are complex numbers such that $\Re e(x)>0$ and $\Re e(y)>0$ and, of course, $\Gamma(x)$ and $B(x, y)$ are complex functions. Since this paper deals with some inequalities involving expressions in $x$ and $y$, we then restrict ourselves for real variables and real functions.

Remark 1.2 The formula (1.8) brings us some interesting results when the conditions $a>0$ and $b-a>0$ are satisfied. For instance, we have the following assertions:

(i) The real-map $z \mapsto{ }_{1} F_{1}(a ; b ; z)$ is strictly increasing and strictly convex on $\mathbb{R}$.

(ii) It follows that ${ }_{1} F_{1}(a ; b ; z) \geq{ }_{1} F_{1}(a ; b ; 0)=1$ for any $z \geq 0$ and, by $(1.9), 0 \leq{ }_{1} F_{1}(a ; b ; z) \leq$ 1 for any $z \leq 0$.

These properties are not obvious from (1.7).

The extension of the beta function from two to $n$ variables has been introduced in the literature $[1,2,6,21,22]$. Let $n \geq 3$ be an integer and let $E_{n-1}$ be the standard ( $\left.n-1\right)$ simplex of $\mathbb{R}^{n-1}$ defined by

$$
E_{n-1}=\left\{\left(t_{1}, \ldots, t_{n-1}\right) \in \mathbb{R}^{n-1}: \sum_{i=1}^{n-1} t_{i} \leq 1 ; t_{i} \geq 0, \text { for } i=1, \ldots, n-1\right\} .
$$

The beta function in $n$ variables $x_{1}, \ldots, x_{n}>0$ is defined by

$$
B\left(x_{1}, \ldots, x_{n}\right)=: \int_{E_{n-1}} \prod_{i=1}^{n} t_{i}^{x_{i}-1} d t_{1} \cdots d t_{n-1}
$$

where $t_{n}=: 1-\sum_{i=1}^{n-1} t_{i}$. Throughout the following, we set $\sigma(x)=: \sum_{i=1}^{n} x_{i}$ for the sake of simplicity. The following formula:

$$
B\left(x_{1}, \ldots, x_{n}\right)=\frac{\prod_{i=1}^{n} \Gamma\left(x_{i}\right)}{\Gamma(\sigma(x))}
$$

holds for any $x_{1}, \ldots, x_{n}>0$. Other properties of the beta function in $n$ variables can be found in the literature. Among them we mention the following [6]:

$$
B\left(x_{1}, \ldots, x_{n}\right)=B\left(x_{\tau(1)}, \ldots, x_{\tau(n)}\right),
$$

where $\tau$ is any permutation of the set $\{1,2, \ldots, n\}$, and

$$
B\left(x_{1}+1, x_{2}, \ldots, x_{n}\right)+\cdots+B\left(x_{1}, \ldots, x_{n-1}, x_{n}+1\right)=B\left(x_{1}, \ldots, x_{n}\right) .
$$

Recently, the authors [22] have extended the previous functions $B(x, y ; a)$ and $B(x, y ; a, b)$ for $n$ variables. For any $x_{1}, \ldots, x_{n}>0, a_{1}, \ldots, a_{n}>0$ and $a>0$, they defined the following extensions:

$$
B\left(x_{1}, \ldots, x_{n} ; a\right)=: \int_{E_{n-1}} \prod_{i=1}^{n} t_{i}^{x_{i}-1} e^{-a / \pi(t)} d t_{1} \cdots d t_{n-1},
$$




$$
B\left(x_{1}, \ldots, x_{n} ; a_{1}, \ldots, a_{n}\right)=: \int_{E_{n-1}} \prod_{i=1}^{n} t_{i}^{x_{i}-1} e^{-a_{i} / t_{i}} d t_{1} \cdots d t_{n-1}
$$

where, as before, $t_{n}=: 1-\sum_{i=1}^{n-1} t_{i}$, and $\pi(t)=: \prod_{i=1}^{n} t_{i}$. For a systematic study of the properties of $B\left(x_{1}, \ldots, x_{n} ; a\right)$, as well as those of $B\left(x_{1}, \ldots, x_{n} ; a_{1}, \ldots, a_{n}\right)$, one can consult [22].

This manuscript will be organized as follows: In Sect. 2 we introduce the generalized gamma function in $n$ variables in a brief and simple setting. Section 3 is focused on introducing the first generalized beta function in $n$ variables, and Sect. 4 displays some inequalities involving this generalized beta function. Section 5 is devoted to introducing a second generalized beta function in $n$ variables, and Sect. 6 discusses some inequalities about this generalized beta function.

\section{Generalized gamma function in $\boldsymbol{n}$ variables}

In this section, we will introduce some extensions of (1.3) and (1.6) from one to $n$ variables. We then state the following definition.

Definition 2.1 Let $x=:\left(x_{1}, \ldots, x_{n}\right) \in(0, \infty)^{n}, \alpha=:\left(\alpha_{1}, \ldots, \alpha_{n}\right) \in(0, \infty)^{n}, \beta=:\left(\beta_{1}, \ldots, \beta_{n}\right) \in$ $(0, \infty)^{n}$, and $p=\left(p_{1}, \ldots, p_{n}\right) \in[0, \infty)^{n}$. We introduce the following:

(i) The extended gamma function in $n$ variables defined by

$$
\boldsymbol{\Gamma}_{p}(x)=: \int_{(0, \infty)^{n}} \prod_{i=1}^{n} t_{i}^{x_{i}-1} e^{-t_{i}} e^{-p_{i} / t_{i}} d t=\prod_{i=1}^{n} \Gamma_{p_{i}}\left(x_{i}\right)
$$

where $d t=: d t_{1} \cdots d t_{n}$ and $\Gamma_{p_{i}}\left(x_{i}\right)$ is defined by (1.3). If $p=0$, we simply write $\Gamma(x)$, i.e.,

$$
\boldsymbol{\Gamma}(x)=: \boldsymbol{\Gamma}_{0}(x)=: \prod_{i=1}^{n} \Gamma\left(x_{i}\right)
$$

(ii) The generalized gamma function in $n$ variables defined through

$$
\Gamma_{p}^{(\alpha, \beta)}(x)=: \prod_{i=1}^{n} \Gamma_{p_{i}}^{\left(\alpha_{i}, \beta_{i}\right)}\left(x_{i}\right)=\int_{(0, \infty)^{n}} \prod_{i=1}^{n} t_{i}^{x_{i}-1}{ }_{1} F_{1}\left(\alpha_{i} ; \beta_{i} ;-t_{i}-\frac{p_{i}}{t_{i}}\right) d t
$$

where $\Gamma_{p_{i}}^{\left(\alpha_{i}, \beta_{i}\right)}\left(x_{i}\right)$ is defined by (1.6). If $p=0$, we simply write $\Gamma^{(\alpha, \beta)}(x)$, i.e.,

$$
\Gamma^{(\alpha, \beta)}(x)=: \prod_{i=1}^{n} \Gamma^{\left(\alpha_{i}, \beta_{i}\right)}\left(x_{i}\right)=\int_{(0, \infty)^{n}} \prod_{i=1}^{n} t_{i}^{x_{i}-1}{ }_{1} F_{1}\left(\alpha_{i} ; \beta_{i} ;-t_{i}\right) d t
$$

Some special cases are worth mentioning, as itemized in the following:

- If $n=1$ then (2.1) and (2.3) are reduced to (1.3) and (1.6), respectively.

- If $\alpha=\beta$ then (2.3) coincides with (2.1).

The equality (2.3) means that the generalized gamma function in $n$ variables $\Gamma_{p}^{(\alpha, \beta)}(x)$ is defined as the product in the usual way of the $n$ components $\Gamma_{p_{i}}^{\left(\alpha_{i}, \beta_{i}\right)}\left(x_{i}\right), 1 \leq i \leq n$. Therefore, the properties of $\Gamma_{p}^{(\alpha, \beta)}(x)$ can be immediately deduced from those of $\Gamma_{p_{i}}^{\left(\alpha_{i}, \beta_{i}\right)}\left(x_{i}\right)$. As an example, the following relationship:

$$
\Gamma_{p}^{(\alpha, \beta)}(x)=\Gamma_{p}^{(\alpha, \beta)}(-x) \prod_{i=1}^{n} p_{i}^{x_{i}}
$$


holds for any $x=:\left(x_{1}, \ldots, x_{n}\right) \in(0, \infty)^{n}, \alpha=:\left(\alpha_{1}, \ldots, \alpha_{n}\right) \in(0, \infty)^{n}, \beta=:\left(\beta_{1}, \ldots, \beta_{n}\right) \in$ $(0, \infty)^{n}$, and $p=\left(p_{1}, \ldots, p_{n}\right) \in(0, \infty)^{n}$.

Remark 2.2 The generalized gamma functions in $n$ variables defined by (2.3) and (2.4) would be of use later. See Corollaries 4.7 and 4.11, Theorem 6.3, Corollary 6.4, and Theorem 6.8.

\section{Generalized beta function of the first kind}

We preserve the same notations as in the previous sections. In the ongoing section, we will introduce a generalized beta function in $n$ variables of the first kind as recited in the following.

Definition 3.1 Let $x=:\left(x_{1}, \ldots, x_{n}\right) \in(0, \infty)^{n}, c, d, q>0$ and $r \geq 0$. The generalized beta function of the first kind is defined by

$$
B_{r}^{(c, d)}(x ; q)=: \int_{E_{n-1}} \prod_{i=1}^{n} t_{i}^{x_{i}-1}{ }_{1} F_{1}\left(c ; d ;-\frac{q}{\pi(t)}-r \frac{\pi(t)}{q}\right) d t,
$$

where we set $d t=: d t_{1} \cdots d t_{n-1}$, and $\pi(t)=: \prod_{i=1}^{n} t_{i}$ with $t_{n}=: 1-\sum_{i=1}^{n-1} t_{i}$. For $r=0$, we set throughout the following:

$$
B^{(c, d)}(x ; q)=: B_{0}^{(c, d)}(x ; q)=: \int_{E_{n-1}} \prod_{i=1}^{n} t_{i}^{x_{i}-1}{ }_{1} F_{1}\left(c ; d ;-\frac{q}{\pi(t)}\right) d t .
$$

Some special cases and a concept of singularity of (3.1) are worth mentioning, such as:

- If $n=2$ and $r=0$ then (3.1) coincides with (1.5).

- If $c=d$ and $r=0$ then (3.1) is exactly (1.14).

- If $r \neq 0$ then (3.1) presents a singularity at $q=0$. Such a singularity has been eliminated in (3.2).

The existence of the integral on the right hand-side of (3.1) is justified by the following result.

Proposition 3.2 Let $x=:\left(x_{1}, \ldots, x_{n}\right) \in(0, \infty)^{n}, r \geq 0$ and $c, d, q>0$, with $d-c>0$. Then $0 \leq B_{r}^{(c, d)}(x ; q) \leq B(x)$ and so, $B_{r}^{(c, d)}(x ; q)$ is well-defined.

Proof By Remark 1.2(ii), we have

$$
0 \leq \prod_{i=1}^{n} t_{i}^{x_{i}-1}{ }_{1} F_{1}\left(c ; d ;-\frac{q}{\pi(t)}-r \frac{\pi(t)}{q}\right) \leq \prod_{i=1}^{n} t_{i}^{x_{i}-1} .
$$

Integrating (3.3) side by side over $t \in E_{n-1}$, with the help of (1.10) and (3.1), we get the desired result.

The basic properties of $B_{r}^{(c, d)}(x ; q)$, analogous to (1.12) and (1.13), are embodied in the following result. 
Proposition 3.3 Let $x, c, d, r$, and $q$ be as previously.

(i) The following relationship holds:

$$
B_{r}^{(c, d)}(x ; q)=B_{r}^{(c, d)}\left(x^{*} ; q\right)
$$

where we set $x^{*}=:\left(x_{\tau(1)}, \ldots, x_{\tau(n)}\right)$ for any permutation $\tau$ of the set $\{1,2, \ldots, n\}$.

(ii) We have

$$
\sum_{j=1}^{n} B_{r}^{(c, d)}\left(x+e_{j} ; q\right)=B_{r}^{(c, d)}(x ; q)
$$

where $\left(e_{1}, \ldots, e_{n}\right)$ refers to the canonical basis of $\mathbb{R}^{n}$.

Proof (i) Let $y=:\left(x_{1}, \ldots, x_{j}, \ldots, x_{k}, \ldots, x_{n}\right)$ and $z=:\left(x_{1}, \ldots, x_{k}, \ldots, x_{j} \ldots, x_{n}\right)$. It is enough to show that $B_{r}^{(c, d)}(y ; q)=B_{r}^{(c, d)}(z ; q)$ for any $j, k$ such that $1 \leq j<k \leq n$. We consider the following change of variables:

$$
t_{1}=u_{1}, \ldots, t_{j}=u_{k}, \ldots, t_{k}=u_{j}, \ldots, t_{n}=u_{n} .
$$

First, it is obvious that $\pi(t)=: t_{1} \cdots t_{n}=u_{1} \cdots u_{n}=: \pi(u)$. Further, it is clear that $\left(t_{1}, \ldots, t_{n}\right) \in$ $E_{n-1}$ if and only if $\left(u_{1}, \ldots, u_{n}\right) \in E_{n-1}$. Moreover, it is easy to see that the absolute value of the Jacobian $J$ of the transformation $\left(t_{1}, \ldots, t_{n}\right) \mapsto\left(u_{1}, \ldots, u_{n}\right)$ defined by (3.4) is given by $|J|=1$. By (3.1), with the standard rules of calculus, we get

$$
\begin{aligned}
B_{r}^{(c, d)}(y ; q) & =\int_{E_{n-1}} \prod_{i=1, i \neq j, k}^{n} t_{i}^{x_{i}-1} t_{j}^{x_{j}-1} t_{k}^{x_{k}-1}{ }_{1} F_{1}\left(c ; d ;-\frac{q}{\pi(t)}-r \frac{\pi(t)}{q}\right) d t \\
& =\int_{E_{n-1}} \prod_{i=1, i \neq j, k}^{n} u_{i}^{x_{i}-1} u_{j}^{x_{k}-1} u_{k}^{x_{j}-1}{ }_{1} F_{1}\left(c ; d ;-\frac{q}{\pi(u)}-r \frac{\pi(u)}{q}\right) d u \\
& =B_{r}^{(c, d)}(z ; q),
\end{aligned}
$$

and hence the desired result.

(ii) By (3.1), we have

$$
\sum_{j=1}^{n} B_{r}^{(c, d)}\left(x+e_{j} ; q\right)=\int_{E_{n-1}} \sum_{j=1}^{n} \prod_{i=1, i \neq j}^{n} t_{i}^{x_{i}-1} t_{j}^{x_{j}} F_{1}\left(c ; d ;-\frac{q}{\pi(t)}-r \frac{\pi(t)}{q}\right) d t
$$

or, equivalently,

$$
\sum_{j=1}^{n} B_{r}^{(c, d)}\left(x+e_{j} ; q\right)=\int_{E_{n-1}} \prod_{i=1}^{n} t_{i}^{x_{i}-1}\left(\sum_{j=1}^{n} t_{j}\right){ }_{1} F_{1}\left(c ; d ;-\frac{q}{\pi(t)}-r \frac{\pi(t)}{q}\right) d t .
$$

Since $\sum_{j=1}^{n} t_{j}=1$, we then get the desired equality, completing the proof.

Another property concerning $B^{(c, d)}(x ; q)$ reads as follows. 
Theorem 3.4 Let $x, c, d$, and $q$ be as above. Assume that $c, d-c>0$. Then we have

$$
B^{(c, d)}(x ; q)=\frac{\Gamma(d)}{\Gamma(c) \Gamma(d-c)} \int_{0}^{1} u^{c-1}(1-u)^{d-c-1} B(x ; q u) d u
$$

where $B(x ; q u)$ is defined by (1.14).

Proof By (3.2) and (1.8), we have

$$
\begin{aligned}
B^{(c, d)}(x ; q) & =\int_{E_{n-1}} \prod_{i=1}^{n} t_{i}^{x_{i}-1}{ }_{1} F_{1}\left(c ; d ;-\frac{q}{\pi(t)}\right) d t \\
& =\frac{\Gamma(d)}{\Gamma(c) \Gamma(d-c)} \int_{E_{n-1}}\left\{\prod_{i=1}^{n} t_{i}^{x_{i}-1} \int_{0}^{1} u^{c-1}(1-u)^{d-c-1} e^{-\frac{q u}{\pi(t)}} d u\right\} d t,
\end{aligned}
$$

where, as before, $d t=d t_{1} \cdots d t_{n-1}$ and $\pi(t)=t_{1} \cdots t_{n}$. By virtue of the uniform convergence of the involved integrals, we can interchange their orders and obtain

$$
B^{(c, d)}(x ; q)=\frac{\Gamma(d)}{\Gamma(c) \Gamma(d-c)} \int_{0}^{1} u^{c-1}(1-u)^{d-c-1}\left(\int_{E_{n-1}} \prod_{i=1}^{n} t_{i}^{x_{i}-1} e^{-\frac{q u}{\pi(t)}} d t\right) d u,
$$

which, when combined with (1.14), immediately implies (3.5).

\section{Inequalities involving $B_{r}^{(c, d)}(x ; q)$}

This section deals with some inequalities involving the beta function of the first kind. Our first main result in this section is recited in the following.

Theorem 4.1 Let $c>0, d-c>0, q>0$, and $r \geq 0$. The following inequality:

$$
\left(B_{r}^{(c, d)}(x+y ; q)\right)^{2} \leq B_{r}^{(c, d)}(2 x ; q) B_{r}^{(c, d)}(2 y ; q)
$$

holds for any $x, y \in(0, \infty)^{n}$. Therefore, the real-valued function $x \mapsto B_{r}^{(c, d)}(x ; q)$ is convex on $(0, \infty)^{n}$.

Proof Let $x=\left(x_{1}, \ldots, x_{n}\right), y=\left(y_{1}, \ldots, y_{n}\right) \in(0, \infty)^{n}$ and set

$$
\omega(t)=:{ }_{1} F_{1}\left(c ; d ;-\frac{q}{\pi(t)}-r \frac{\pi(t)}{q}\right)
$$

By Remark 1.2(ii), we have $\omega(t) \geq 0$. Then we can write

$$
\left(B_{r}^{(c, d)}(x+y ; q)\right)^{2}=\left(\int_{E_{n-1}}\left(\prod_{i=1}^{n} t_{i}^{x_{i}-1 / 2}(\omega(t))^{1 / 2}\right)\left(\prod_{i=1}^{n} t_{i}^{y_{i}-1 / 2}(\omega(t))^{1 / 2}\right) d t\right)^{2}
$$

This, with the Cauchy-Schwartz inequality for integrals, yields

$$
\left(B_{r}^{(c, d)}(x+y ; q)\right)^{2} \leq \int_{E_{n-1}} \prod_{i=1}^{n} t_{i}^{2 x_{i}-1} \omega(t) d t \int_{E_{n-1}} \prod_{i=1}^{n} t_{i}^{2 y_{i}-1} \omega(t) d t
$$


Hence (4.1). Now, let us remark that (4.1) is equivalent to

$$
B_{r}^{(c, d)}\left(\frac{x+y}{2} ; q\right) \leq\left(B_{r}^{(c, d)}(x ; q) B_{r}^{(c, d)}(y ; q)\right)^{1 / 2} \text {. }
$$

This, with the arithmetic-geometric mean inequality $\sqrt{a b} \leq \frac{1}{2} a+\frac{1}{2} b$ valid for any $a, b \geq 0$, implies that

$$
B_{r}^{(c, d)}\left(\frac{x+y}{2} ; q\right) \leq \frac{1}{2} B_{r}^{(c, d)}(x ; q)+\frac{1}{2} B_{r}^{(c, d)}(y ; q) .
$$

We then deduce that $x \mapsto B_{r}^{(c, d)}(x ; q)$ is mid-convex. This, with the fact that $x \mapsto B_{r}^{(c, d)}(x ; q)$ is continuous, ensures that $x \mapsto B_{r}^{(c, d)}(x ; q)$ is convex. The proof is finished.

To giving more results, we need the following lemma.

Lemma 4.2 Let $a, b>0$. Then the real-valued function $z \mapsto{ }_{1} F_{1}(a ; b ; z)$ is differentiable on $\mathbb{R}$ and we have

$$
\frac{d}{d z}{ }_{1} F_{1}(a ; b ; z)=\frac{a}{b}{ }_{1} F_{1}(a+1 ; b+1 ; z) .
$$

In particular, one has

$$
\left.\frac{d}{d z}{ }_{1} F_{1}(a ; b ; z)\right|_{z=0}=\frac{a}{b} .
$$

Proof For the proof of (4.2), see [18]. Since ${ }_{1} F_{1}(a ; b ; 0)=1$ for any $a, b>0$, we then deduce (4.3).

Now, we may state the following result.

Theorem 4.3 Let $c>0, d-c>0, q>0$, and $r \geq 0$. Then the following inequalities:

$$
B^{(c, d)}(x ; q)-\frac{c}{d} \frac{r}{q} B^{(c+1, d+1)}(x+e ; q) \leq B_{r}^{(c, d)}(x ; q) \leq B^{(c, d)}(x ; q) \leq B(x)
$$

hold true for all $x \in(0, \infty)^{n}$, where we set $e=:(1,1, \ldots, 1)$.

Proof By (3.1) and (3.2), with the help of Remark 1.2(ii), we deduce that $B_{r}^{(c, d)}(x ; q) \leq$ $B^{(c, d)}(x ; q) \leq B(x)$. We now prove the inequality

$$
B^{(c, d)}(x ; q)-\frac{c}{d} \frac{r}{q} B^{(c+1, d+1)}(x+e ; q) \leq B_{r}^{(c, d)}(x ; q) .
$$

According to Remark 1.2(i), the map $z \mapsto{ }_{1} F_{1}(a ; b ; z)$ is convex on $\mathbb{R}$, for fixed $a, b-a>0$. Let us recall that if $f: \mathbb{R} \rightarrow \mathbb{R}$ is a convex function, differentiable at $z_{0}$, then we have $f(z) \geq$ $f\left(z_{0}\right)+\left(z-z_{0}\right) f^{\prime}\left(z_{0}\right)$ for all $z \in \mathbb{R}$. Applying this with $f(z)=:{ }_{1} F_{1}(c ; d ; z)$, and utilizing (4.2), we obtain

$$
\begin{aligned}
& { }_{1} F_{1}\left(c ; d ;-\frac{q}{\pi(t)}-r \frac{\pi(t)}{q}\right) \\
& \quad \geq{ }_{1} F_{1}\left(c ; d ;-\frac{q}{\pi(t)}\right)-\frac{c}{d} \frac{r}{q} \pi(t){ }_{1} F_{1}\left(c+1 ; d+1 ;-\frac{q}{\pi(t)}\right) .
\end{aligned}
$$


Multiplying (4.6) by $\prod_{i=1}^{n} t^{x_{i}-1}$ and integrating side by side over $t \in E_{n-1}$, with the help of (3.1), (3.2), and the fact that $\pi(t)=: \prod_{i=1}^{n} t_{i}$, we get (4.5). The proof is complete.

It is worth mentioning that (4.5) gives the converse of $B_{r}^{(c, d)}(x ; q) \leq B^{(c, d)}(x ; q)$. The following result concerns the converse of the inequality $B_{r}^{(c, d)}(x ; q) \leq B(x)$.

Proposition 4.4 Let $c>0, d-c>0, q>0$, and $r \geq 0$. For any $x \in(1, \infty)^{n}$, we have

$$
B_{r}^{(c, d)}(x ; q) \geq B(x)-\frac{c}{d} q B(x-e)-\frac{c}{d} \frac{r}{q} B(x+e),
$$

where, as previously, $e=:(1,1, \ldots, 1)$.

Proof By the same arguments as in the proof of Theorem 4.3 and by using (4.3), we get

$$
{ }_{1} F_{1}\left(c ; d ;-\frac{q}{\pi(t)}-r \frac{\pi(t)}{q}\right) \geq{ }_{1} F_{1}(c ; d ; 0)+\frac{c}{d}\left(-\frac{q}{\pi(t)}-r \frac{\pi(t)}{q}\right) .
$$

Multiplying (4.8) by $\prod_{i=1}^{n} t^{x_{i}-1}$, our conclusion then would be similar to that in Theorem 4.3. The details are simple and therefore omitted here.

In order to give more results, we need to prove the lemma below. Recall that, with $t=$ : $\left(t_{1}, \ldots, t_{n-1}\right)$, we have

$$
\pi(t)=: t_{1} \cdots t_{n-1} t_{n}=t_{1} \cdots t_{n-1}\left(1-t_{1}-\cdots-t_{n-1}\right) .
$$

Lemma 4.5 With the above we have

$$
\sup _{t \in E_{n-1}} \pi(t)=n^{-n}
$$

Proof We will prove this lemma following two different ways.

First method. Let $a_{1}, a_{2}, \ldots, a_{n}$ be $n$ positive real numbers. The following inequality:

$$
\sqrt[n]{a_{1} a_{2} \cdots a_{n}} \leq \frac{a_{1}+a_{2}+\cdots+a_{n}}{n}
$$

is well known as the arithmetic-geometric mean inequality in $n$ variables. Letting $a_{1}=$ $t_{1}, a_{2}=t_{2}, \ldots, a_{n-1}=t_{n-1}, a_{n}=t_{n}=: 1-t_{1}-\cdots-t_{n-1}$ in (4.9), we obtain $\sqrt[n]{\pi(t)} \leq \frac{1}{n}$, i.e., $\pi(t) \leq n^{-n}$ for any $t \in E_{n-1}$. It is clear that the inequality $\pi(t) \leq n^{-n}$ becomes an equality for $\left(t_{1}, t_{2}, \ldots, t_{n-1}\right)=(1 / n, 1 / n, \ldots, 1 / n) \in E_{n-1}$. The desired result follows.

Second method. Let us first remark that the simplex $E_{n-1}$ is a compact set of $\mathbb{R}^{n-1}$ and the real-valued function $t \mapsto \pi(t)$ is continuous on $E_{n-1}$. So, the supremum of $\pi(t)$ on $E_{n-1}$ exists and it is achieved, i.e., $\max _{t \in E_{n-1}} \pi(t)=\pi(v)$ for some $v \in E_{n-1}$. Further, it is well known that the set of all extremal points of $E_{n-1}$, as a convex compact of $\mathbb{R}^{n-1}$, is given by $\operatorname{Ext}\left(E_{n-1}\right)=\left\{e_{0}, e_{1}, \ldots, e_{n-1}\right\}$, where $e_{0}$ is the zero-vector of $\mathbb{R}^{n-1}$ and $\left(e_{1}, \ldots, e_{n-1}\right)$ is the canonical basis of $\mathbb{R}^{n-1}$. It is obvious that $\pi(t)=0$ for any $t \in \operatorname{Ext}\left(E_{n-1}\right)$. This, plus the equality $\inf _{t \in E_{n-1}} \pi(t)=0$, ensures that $v$ will be a critical point of the differentiable 
function $t \mapsto \pi(t)$. We will then find the critical points of $t \mapsto \pi(t)$. Simple computation leads to

$$
\frac{\partial \pi}{\partial t_{i}}(t)=\left(\prod_{j=1, j \neq i}^{n-1} t_{j}\right)\left(1-\sum_{j=1, j \neq i}^{n-1} t_{j}-2 t_{i}\right), \quad 1 \leq i \leq n-1 .
$$

Then we get

$$
\frac{\partial \pi}{\partial t_{i}}(t)=0 \Longleftrightarrow 1-\sum_{j=1, j \neq i}^{n-1} t_{j}-2 t_{i}=0, \quad 1 \leq i \leq n-1 .
$$

We have to solve the $(n-1)$-equations, namely

$$
\sum_{j=1, j \neq i}^{n-1} t_{j}+2 t_{i}=1, \quad 1 \leq i \leq n-1
$$

in the unknown $\left(t_{1}, \ldots, t_{n-1}\right)$. Thus, (4.10) is a linear system whose matrix is $M=I+P$ where $I$ is the identity matrix and $P=\left(p_{i j}\right)$ is such that $p_{i j}=1$ for any $i, j=1, \ldots, n-1$. If we denote the euclidean inner product of $\mathbb{R}^{n-1}$ by $\langle\cdot, \cdot\rangle$, then it is easy to check that $\langle P w, w\rangle=\left(w_{1}+\right.$ $\left.\cdots+w_{n-1}\right)^{2} \geq 0$ for any $w=\left(w_{1}, \ldots, w_{n-1}\right) \in \mathbb{R}^{n-1}$. We then deduce that $\langle M w, w\rangle \geq\|w\|^{2}$ for any $w \in \mathbb{R}^{n-1}$, where $\|\cdot\|$ refers to the euclidean norm of $\mathbb{R}^{n-1}$. It follows that the matrix $M$, which is clearly symmetric, is positive definite and so it is invertible. We conclude that the linear system (4.10) has one and only one solution. Otherwise, (4.10) is symmetric in $t_{1}, \ldots, t_{n-1}$ and therefore its unique solution satisfies $t_{1}=t_{2}=\cdots=t_{n-1}=: \tau$. Substituting this into (4.10), we get $n \tau=1$ and so the unique solution of $(4.10)$ is $(1 / n, 1 / n, \ldots, 1 / n)$. In conclusion, $t \mapsto \pi(t)$ has one and only one critical point $v$, and we then conclude that $v=$ $(1 / n, \ldots, 1 / n)$. A simple computation leads to $\pi(v)=n^{-n}$, and the desired result follows.

We are now in a position to state the following result which gives a refinement of the inequality $B_{r}^{(c, d)}(x ; q) \leq B(x)$.

Theorem 4.6 Let $c>0, d-c>0, q>0, r \geq 0$, and $x \in(0, \infty)^{n}$. Assume that further $q \geq \sqrt{r}$. Then we have

$$
\frac{B_{r}^{(c, d)}(x ; q)}{B(x)} \leq{ }_{1} F_{1}\left(c ; d ;-q n^{n}-\frac{r}{q n^{n}}\right) \leq{ }_{1} F_{1}(c ; d ;-2 \sqrt{r}) \leq 1
$$

where $B(x)$ is defined by (1.10).

Proof The inequalities

$$
{ }_{1} F_{1}\left(c ; d ;-q n^{n}-\frac{r}{q n^{n}}\right) \leq{ }_{1} F_{1}(c ; d ;-2 \sqrt{r}) \leq 1
$$

are immediate since it is easy to check that

$$
-q n^{n}-\frac{r}{q n^{n}} \leq-2 \sqrt{r} \leq 0, \quad{ }_{1} F_{1}(c ; d ; 0)=1
$$


and the real-valued function $z \mapsto{ }_{1} F_{1}(c ; d ; z)$ is increasing. We will now prove the inequality

$$
\frac{B_{r}^{(c, d)}(x ; q)}{B(x)} \leq{ }_{1} F_{1}\left(c ; d ;-q n^{n}-\frac{r}{q n^{n}}\right)
$$

By (3.1), we deduce that

$$
B_{r}^{(c, d)}(x ; q) \leq B(x) \sup _{t \in E_{n-1}} F_{1}\left(c ; d ;-\frac{q}{\pi(t)}-r \frac{\pi(t)}{q}\right)
$$

provided that the involved supremum exists. By (1.8), we have

$$
\begin{aligned}
{ }_{1} & F_{1}\left(c ; d ;-\frac{q}{\pi(t)}-r \frac{\pi(t)}{q}\right) \\
& =\frac{\Gamma(d)}{\Gamma(c) \Gamma(d-c)} \int_{0}^{1} u^{c-1}(1-u)^{d-c-1} \exp u\left(-\frac{q}{\pi(t)}-r \frac{\pi(t)}{q}\right) d u .
\end{aligned}
$$

It is then enough to find an upper bound of $t \mapsto-\frac{q}{\pi(t)}-r \frac{\pi(t)}{q}$, since $u \in[0,1]$ and the exponential function is increasing. By the standard techniques of real analysis, it is easy to see that if $q \geq \sqrt{r}$ and $0<\rho<1$ then

$$
\sup _{0 \leq s \leq \rho}\left(-\frac{q}{s}-r \frac{s}{q}\right)=-\frac{q}{\rho}-r \frac{\rho}{q}
$$

Otherwise, Lemma 4.5 asserts that $\pi(t) \leq n^{-n}$, and so we can write

$$
E_{n-1} \subset\left\{\left(t_{1}, \ldots, t_{n-1}\right): 0 \leq \pi(t) \leq n^{-n}\right\}=: F .
$$

This, with (4.15), implies that

$$
\begin{aligned}
\sup _{t \in E_{n-1}}\left(-\frac{q}{\pi(t)}-r \frac{\pi(t)}{q}\right) & \leq \sup _{t \in F}\left(-\frac{q}{\pi(t)}-r \frac{\pi(t)}{q}\right) \\
& =\sup _{0 \leq s \leq n^{-n}}\left(-\frac{q}{s}-r \frac{s}{q}\right)=-q n^{n}-\frac{r}{q n^{n}} .
\end{aligned}
$$

Substituting this into (4.14) and then into (4.13), we get (4.12). The proof is finished.

From the previous theorem, we can deduce the following result.

Corollary 4.7 With the hypotheses of Theorem 4.6, for any $u>0$, we have:

$$
\int_{0}^{\infty} q^{u-1} B^{(c, d}(x ; q) d q \leq B(x) n^{-n u} \Gamma^{(c, d)}(u),
$$

where $\Gamma^{(c, d)}(u)$ is defined by (2.4). In particular,

$$
\int_{0}^{\infty} B^{(c, d}(x ; q) d q \leq B(x) n^{-n} \Gamma^{(c, d)}(1) .
$$


Proof Taking $r=0$ in (4.11), we get

$$
B^{(c, d)}(x ; q) \leq B(x)_{1} F_{1}\left(c ; d ;-q n^{n}\right) .
$$

Multiplying (4.17) by $q^{u-1}$ and integrating side by side over $q \in(0, \infty)$, we obtain (4.16) after a simple change of variable.

Now we will state a result which gives a lower bound of $B_{r}^{(c, d)}(x ; q)$.

Theorem 4.8 Let $c>0, d-c>0, q>0, r \geq 0$, and $x \in(0, \infty)^{n}$. Then we have

$$
\frac{B_{r}^{(c, d)}(x ; q)}{B(x ; q)} \geq{ }_{1} F_{1}\left(d-c ; d ; q n^{n}\right) \exp \left(-\frac{r}{q n^{n}}\right)
$$

where $B(x ; q)$ is defined by $(1.14)$.

Proof By (1.9), we can write

$$
B_{r}^{(c, d)}(x ; q)=\int_{E_{n-1}} \prod_{i=1}^{n} t_{i}^{x_{i}-1} \exp \left(-\frac{q}{\pi(t)}-r \frac{\pi(t)}{q}\right){ }_{1} F_{1}\left(d-c ; d ; \frac{q}{\pi(t)}+r \frac{\pi(t)}{q}\right) d t
$$

or, equivalently,

$$
B_{r}^{(c, d)}(x ; q)=\int_{E_{n-1}}\left(\prod_{i=1}^{n} t_{i}^{x_{i}-1} e^{-\frac{q}{\pi(t)}}\right) e^{-\frac{r \pi(t)}{q}}{ }_{1} F_{1}\left(d-c ; d ; \frac{q}{\pi(t)}+r \frac{\pi(t)}{q}\right) d t .
$$

Now, by Lemma 4.5, we deduce that

$$
e^{-\frac{r \pi(t)}{q}} \geq e^{-\frac{r}{q n^{n}}} \quad \text { and } \quad \frac{q}{\pi(t)}+r \frac{\pi(t)}{q} \geq \frac{q}{\pi(t)} \geq q n^{n}
$$

Substituting this into (4.19) and taking into account that the real-valued function $z \mapsto$ ${ }_{1} F_{1}(a ; b ; z)$ is increasing, with the help of (1.14), we get (4.18), and the proof is finished.

We have the following result as well.

Corollary 4.9 With the hypotheses of Theorem 4.8, for any $u>0$, we have:

$$
\int_{0}^{\infty} r^{u-1} B_{r}^{(c, d}(x ; q) d r \geq\left(q n^{n}\right)^{u} \Gamma(u) B(x ; q)_{1} F_{1}\left(d-c ; d ; q n^{n}\right) .
$$

In particular, if $u=1$, one has

$$
\int_{0}^{\infty} B_{r}^{(c, d}(x ; q) d r \geq q n^{n} B(x ; q)_{1} F_{1}\left(d-c ; d ; q n^{n}\right) .
$$

Proof Multiplying (4.18) by $r^{u-1}$ and then integrating over $r \in(0, \infty)$, we get

$$
\int_{0}^{\infty} r^{u-1} B_{r}^{(c, d}(x ; q) d r \geq B(x ; q)_{1} F_{1}\left(d-c ; d ; q n^{n}\right) \int_{0}^{\infty} r^{u-1} e^{-\frac{r}{q n^{n}}} d r .
$$


Making the change of variable $t=\frac{r}{q n^{n}}$ in (4.22), with some simple manipulations, we get (4.20) and then (4.21).

The following result may be stated as well.

Theorem 4.10 Let $c>0, d-c>0, q>0, r \geq 0$, and $x \in(0, \infty)^{n}$. Then we have

$$
\frac{B_{r}^{(c, d)}(x ; q)}{B(x ; q)} \geq{ }_{1} F_{1}\left(c ; d ;-\frac{r}{q n^{n}}\right) .
$$

Proof Again by (1.8), we have

$$
\begin{aligned}
& { }_{1} F_{1}\left(c ; d ;-\frac{q}{\pi(t)}-r \frac{\pi(t)}{q}\right) \\
& \quad=\frac{\Gamma(d)}{\Gamma(c) \Gamma(d-c)} \int_{0}^{1} u^{c-1}(1-u)^{d-c-1} \exp \left(-u \frac{q}{\pi(t)}\right) \exp \left(-u r \frac{\pi(t)}{q}\right) d u .
\end{aligned}
$$

By Lemma 4.5, we have, for any $u \in[0,1]$,

$$
\exp \left(-u \frac{q}{\pi(t)}\right) \geq \exp \left(-\frac{q}{\pi(t)}\right) \quad \text { and } \quad \exp \left(-u r \frac{\pi(t)}{q}\right) \geq \exp \left(-u \frac{r}{q n^{n}}\right)
$$

Substituting these into the following formula:

$$
B_{r}^{(c, d)}(x ; q)=\int_{E_{n-1}} \prod_{i=1}^{n} t_{i}^{x_{i}-1}{ }_{1} F_{1}\left(c ; d ;-\frac{q}{\pi(t)}-r \frac{\pi(t)}{q}\right) d t
$$

with an argument of uniform convergence of the involved integrals, we get

$$
\begin{aligned}
B_{r}^{(c, d)}(x ; q) \geq & \frac{\Gamma(d)}{\Gamma(c) \Gamma(d-c)} \int_{0}^{1} u^{c-1}(1-u)^{d-c-1} \exp \left(-u \frac{r}{q n^{n}}\right) d u \\
& \times \int_{E_{n-1}} \prod_{i=1}^{n} t_{i}^{x_{i}-1} \exp \left(-\frac{q}{\pi(t)}\right) d t .
\end{aligned}
$$

Whence (4.23). The proof is finished.

Finally, we state the following corollary.

Corollary 4.11 With the same hypotheses as in Theorem 4.10, for any $u>0$, we have:

$$
\int_{0}^{\infty} r^{u-1} B_{r}^{(c, d}(x ; q) d r \geq\left(q n^{n}\right)^{u} B(x ; q) \Gamma^{(c, d)}(u)
$$

where $\Gamma^{(c, d)}(u)$ is defined by (2.4). In particular, if taking $u=1$, one has

$$
\int_{0}^{\infty} B_{r}^{(c, d}(x ; q) d r \geq q n^{n} B(x ; q) \Gamma^{(c, d)}(1)
$$


Proof If we multiply (4.23) by $r^{u-1}$ and then integrate over $r \in(0, \infty)$, we get

$$
\int_{0}^{\infty} r^{u-1} B_{r}^{(c, d}(x ; q) d r \geq B(x ; q) \int_{0}^{\infty} r^{u-1}{ }_{1} F_{1}\left(c ; d ;-\frac{r}{q n^{n}}\right) d r .
$$

Making the change of variable $r=q n^{n} t$ in (4.25), we get

$$
\int_{0}^{\infty} r^{u-1} B_{r}^{(c, d}(x ; q) d r \geq\left(q n^{n}\right)^{u} B(x ; q) \int_{0}^{\infty} t^{u-1}{ }_{1} F_{1}(c ; d ;-t) d t .
$$

Hence (4.24).

\section{Generalized beta function of the second kind}

This section deals with another generalized beta function in $n$ variables. In what follows, we need to use the following notation:

$$
\begin{array}{ll}
x=:\left(x_{1}, \ldots, x_{n}\right), & a=:\left(a_{1}, \ldots, a_{n}\right), \quad \alpha=:\left(\alpha_{1}, \ldots, \alpha_{n}\right), \\
\beta=:\left(\beta_{1}, \ldots, \beta_{n}\right), & p=:\left(p_{1}, \ldots, p_{n}\right) .
\end{array}
$$

The central definition of this section is given in what follows.

Definition 5.1 Let $x, \alpha, \beta, a \in(0, \infty)^{n}$, and $p \in[0, \infty)^{n}$. The generalized beta function of the second kind is defined by

$$
B_{p}^{(\alpha, \beta)}(x ; a)=: \int_{E_{n-1}} \prod_{i=1}^{n} t_{i}^{x_{i}-1}{ }_{1} F_{1}\left(\alpha_{i} ; \beta_{i} ;-\frac{a_{i}}{t_{i}}-p_{i} \frac{t_{i}}{a_{i}}\right) d t
$$

where we set $d t=: d t_{1} \cdots d t_{n-1}$ and $t_{n}=: 1-\sum_{i=1}^{n-1} t_{i}$. If $p=0$, we simply write $B^{(\alpha, \beta)}(x ; a)$, i.e.,

$$
B^{(\alpha, \beta)}(x ; a)=: \int_{E_{n-1}} \prod_{i=1}^{n} t_{i}^{x_{i}-1}{ }_{1} F_{1}\left(\alpha_{i} ; \beta_{i} ;-\frac{a_{i}}{t_{i}}\right) d t
$$

Some special cases are included in the previous definition:

- If $\alpha=\beta$ and $n=2$ then (5.2) coincides with (1.4).

- If $\alpha=\beta$ and $p=0$ then (5.1) is exactly (1.15).

- If $\alpha=\beta, p=0$ and $a \rightarrow 0$ then (5.1) and (5.2) are both reduced to (1.10).

As for the generalized beta function of the first kind, one can easily check that the inequalities $0 \leq B_{p}^{(\alpha, \beta)}(x ; a) \leq B(x)$ hold for any $x, \alpha, \beta-\alpha, a \in(0, \infty)^{n}$ and $p \in[0, \infty)^{n}$. This justifies the existence of the integral defining $B_{p}^{(\alpha, \beta)}(x ; a)$ through (5.1).

The basic properties of $B_{p}^{(\alpha, \beta)}(x ; a)$, analogous to those of Proposition 3.3, are embodied in the following result.

Proposition 5.2 Let $x, \alpha, \beta, a, p$ be as previously.

(i) We have the following relationship:

$$
B_{p}^{(\alpha, \beta)}(x ; a)=B_{p^{*}}^{\left(\alpha^{*}, \beta^{*}\right)}\left(x^{*} ; a^{*}\right),
$$


where $\tau$ is any permutation of the set $\{1,2, \ldots, n\}$ and $p^{*}=:\left(p_{\tau(1)}, \ldots, p_{\tau(n)}\right)$, with similar settings for $\alpha^{*}, \beta^{*}, x^{*}, a^{*}$.

(ii) The following relation holds:

$$
\sum_{i=1}^{n} B_{p}^{(\alpha, \beta)}\left(x+e_{i} ; a\right)=B_{p}^{(\alpha, \beta)}(x ; a)
$$

where $\left(e_{1}, \ldots, e_{n}\right)$ refers to the canonical basis of $\mathbb{R}^{n}$.

Proof Similar to that of Proposition 3.3. The details are left for the reader.

Following the uniform convergence of the series (1.7), we can interchange in (5.1) the series and the integral. This shows that (5.1) is well defined, i.e., the involved integral is convergent. Further, such an integral is uniformly convergent in any compact set included in the interior of $E_{n-1}$. This implies that we can take limit and differentiation under the integral sign of (5.1). In particular, we have

$$
\lim _{p \rightarrow 0} B_{p}^{(\alpha, \beta)}(x ; a)=B^{(\alpha, \beta)}(x ; a)
$$

where $B^{(\alpha, \beta)}(x ; a)$ is defined by (5.2).

The following result justifies that $B_{p}^{(\alpha ; \beta)}(x ; a)$ is a generalization of the extended beta function $B(x ; a)$ in $n$ variables defined by (1.15).

Proposition 5.3 For any $\alpha, x, a \in(0, \infty)^{n}$, we have

$$
B(x ; a)=\lim _{p \rightarrow 0} B_{p}^{(\alpha ; \alpha)}(x ; a)=: B^{(\alpha, \alpha)}(x ; a) .
$$

Proof By (1.7), with the help of (5.3) and (5.2), we have for any $i=1,2, \ldots, n$,

$$
{ }_{1} F_{1}\left(\alpha_{i} ; \alpha_{i} ;-\frac{a_{i}}{t_{i}}\right)=\sum_{m=0}^{\infty} \frac{\left(-a_{i} / t_{i}\right)^{m}}{m !}=e^{-a_{i} / t_{i}}
$$

This, with (1.15) and (5.1), yields the desired result.

\section{Inequalities involving $B_{p}^{(\alpha, \beta)}(x ; a)$}

In this section, we will discuss some inequalities involving the generalized beta function of the second kind. We begin by stating the following result.

Theorem 6.1 Let $a, \alpha, \beta-\alpha \in(0, \infty)^{n}$, and $p \in[0, \infty)^{n}$. Then the inequality

$$
\left(B_{p}^{(\alpha, \beta)}(x+y ; a)\right)^{2} \leq B_{p}^{(\alpha, \beta)}(2 x ; a) B_{p}^{(\alpha, \beta)}(2 y ; a)
$$

holds for any $x, y \in(0, \infty)^{n}$. Thus, the real-valued map $x \mapsto B_{p}^{(\alpha, \beta)}(x ; a)$ is convex on $(0, \infty)^{n}$.

Proof Using similar arguments, it is analogous to that of Theorem 4.1. We left to the reader the routine task for formulating this proof in a detailed manner.

We now state the following result which gives an upper bound of $B_{p}^{(\alpha, \beta)}(x ; a)$. 
Theorem 6.2 Let $x, a, \alpha, \beta-\alpha \in(0, \infty)^{n}$, and $p \in[0, \infty)^{n}$. Then

$$
\frac{B_{p}^{(\alpha, \beta)}(x ; a)}{B(x)} \leq \prod_{i=1}^{n} e^{-a_{i}}{ }_{1} F_{1}\left(\beta_{i}-\alpha_{i} ; \beta_{i} ; a_{i}+\frac{p_{i}}{a_{i}}\right)
$$

where $B(x)$ is defined by (1.10).

Proof Since the real-valued function $z \mapsto{ }_{1} F_{1}(a ; b ; z)$ is increasing and $0<t_{i} \leq 1$ for any $i=1, \ldots, n$, we have

$$
B_{p}^{(\alpha, \beta)}(x ; a) \leq \int_{E_{n-1}} \prod_{i=1}^{n} t_{i}^{x_{i}-1}{ }_{1} F_{1}\left(\alpha_{i} ; \beta_{i} ;-a_{i}-p_{i} \frac{t_{i}}{a_{i}}\right) d t
$$

This, with (1.9), implies that

$$
B_{p}^{(\alpha, \beta)}(x ; a) \leq \int_{E_{n-1}} \prod_{i=1}^{n} t_{i}^{x_{i}-1} \exp \left(-a_{i}-p_{i} \frac{t_{i}}{a_{i}}\right){ }_{1} F_{1}\left(\beta_{i}-\alpha_{i} ; \beta_{i} ; a_{i}+p_{i} \frac{t_{i}}{a_{i}}\right) d t .
$$

Again with $0<t_{i} \leq 1$ for $i=1, \ldots, n$, it is clear that

$$
\exp \left(-a_{i}-p_{i} \frac{t_{i}}{a_{i}}\right) \leq e^{-a_{i}} \text { and } a_{i}+p_{i} \frac{t_{i}}{a_{i}} \leq a_{i}+\frac{p_{i}}{a_{i}} .
$$

Substituting (6.3) in (6.2), and using again the fact that $z \mapsto{ }_{1} F_{1}(a ; b ; z)$ is increasing, we get (6.1), and the proof is finished.

We have the following result as well.

Theorem 6.3 Let $x, \alpha, \beta-\alpha \in(0, \infty)^{n}$, and $p \in[0, \infty)^{n}$. For any $u=:\left(u_{1}, \ldots, u_{n}\right) \in(0, \infty)^{n}$, there holds:

$$
\int_{(0, \infty)^{n}} \prod_{i=1}^{n} a_{i}^{u_{i}-1} e^{\frac{p_{i}}{a_{i}}} B_{p}^{(\alpha, \beta)}(x ; a) d a \leq B(x) \Gamma_{p}^{(\alpha, \beta)}(u),
$$

where $d a=: d a_{1} \cdots d a_{n}$ and $\Gamma_{p}^{(\alpha, \beta)}(u)$ is defined by (2.3).

Proof By (1.9), (6.1) can be written as follows:

$$
B_{p}^{(\alpha, \beta)}(x ; a) \leq B(x) \prod_{i=1}^{n} e^{-\frac{p_{i}}{a_{i}}} F_{1}\left(\alpha_{i} ; \beta_{i} ;-a_{i}-\frac{p_{i}}{a_{i}}\right)
$$

or, equivalently,

$$
\prod_{i=1}^{n} e^{\frac{p_{i}}{a_{i}}} B_{p}^{(\alpha, \beta)}(x ; a) \leq B(x)_{1} F_{1}\left(\alpha_{i} ; \beta_{i} ;-a_{i}-\frac{p_{i}}{a_{i}}\right)
$$

Multiplying (6.5) by $\prod_{i=1}^{n} a_{i}^{u_{i}-1}$ and then integrating with respect to $a=:\left(a_{1}, \ldots, a_{n}\right)$ over $(0, \infty)^{n}$, with the help of (2.3), we get (6.4). The proof is finished. 
The following corollary tells us that the map $a \mapsto B_{p}^{(\alpha, \beta)}(x ; a)$ is integrable on $(0, \infty)^{n}$ provided that $\Gamma_{p}^{(\alpha, \beta)}(e)<\infty$.

Corollary 6.4 Let $x, \alpha, \beta, p$ be as in Theorem 6.3. Then we have

$$
(0 \leq) \int_{(0, \infty)^{n}} B_{p}^{(\alpha, \beta)}(x ; a) d a \leq \int_{(0, \infty)^{n}} e^{\frac{p_{i}}{a_{i}}} B_{p}^{(\alpha, \beta)}(x ; a) d a \leq B(x) \Gamma_{p}^{(\alpha, \beta)}(e) .
$$

Proof Taking $u=e=:(1,1, \ldots, 1)$ in (6.4), we get the right-hand side inequality of (6.6). The left-hand side inequality of (6.6) is immediate, since $e^{p_{i} / a_{i}} \geq 1$ and $B_{p}^{(\alpha, \beta)}(x ; a) \geq 0$.

Now, we state the following result which concerns a lower bound of $B_{p}^{(\alpha, \beta)}(x ; a)$.

Theorem 6.5 Let $x, a, \alpha, \beta-\alpha \in(0, \infty)^{n}$, and $p \in[0, \infty)^{n}$. Then we have

$$
\frac{B_{p}^{(\alpha, \beta)}(x ; a)}{B(x ; a)} \geq \prod_{i=1}^{n} e^{-\frac{p_{i}}{a_{i}}}{ }_{1} F_{1}\left(\beta_{i}-\alpha_{i} ; \beta_{i} ; m_{i}\right)
$$

where $B(x ; a)$ is defined by $(1.15)$ and $m_{i}=\max \left(2 \sqrt{p_{i}}, a_{i}\right)$. If, moreover, $a_{i} \geq \sqrt{p_{i}}$ for any $i=1, \ldots, n$, then (6.7) can be refined as follows:

$$
\frac{B_{p}^{(\alpha, \beta)}(x ; a)}{B(x ; a)} \geq \prod_{i=1}^{n} e^{-\frac{p_{i}}{a_{i}}}{ }_{1} F_{1}\left(\beta_{i}-\alpha_{i} ; \beta_{i} ; a_{i}+\frac{p_{i}}{a_{i}}\right) .
$$

Proof By (1.9), (5.1) can be written as follows:

$$
B_{p}^{(\alpha, \beta)}(x ; a)=\int_{E_{n-1}}\left(\prod_{i=1}^{n} t_{i}^{x_{i}-1} e^{-\frac{a_{i}}{t_{i}}}\right) e^{-p_{i} \frac{t_{i}}{a_{i}}}{ }_{1} F_{1}\left(\beta_{i}-\alpha_{i} ; \beta_{i} ; \frac{a_{i}}{t_{i}}+p_{i} \frac{t_{i}}{a_{i}}\right) d t .
$$

It is easy to see that $\frac{a_{i}}{t_{i}}+p_{i} \frac{t_{i}}{a_{i}} \geq 2 \sqrt{p_{i}}$. Further, since $0<t_{i}<1$ for any $i=1, \ldots, n$ and the real-valued map $z \mapsto{ }_{1} F_{1}(a ; b ; z)$ is increasing, we have

$$
e^{-p_{i} \frac{t_{i}}{a_{i}}} \geq e^{-\frac{p_{i}}{a_{i}}} \quad \text { and } \quad{ }_{1} F_{1}\left(\beta_{i}-\alpha_{i} ; \beta_{i} ; \frac{a_{i}}{t_{i}}+p_{i} \frac{t_{i}}{a_{i}}\right) \geq{ }_{1} F_{1}\left(\beta_{i}-\alpha_{i} ; \beta_{i} ; \max \left(2 \sqrt{p_{i}}, a_{i}\right)\right) .
$$

Substituting these into (6.9), with the help of (1.15), we get (6.7).

Now let us remark that we have

$$
(a, p>0 \text { with } a \geq \sqrt{p}) \Longrightarrow \inf _{0<t<1}\left(\frac{a}{t}+p \frac{t}{a}\right)=a+\frac{p}{a} .
$$

Hence (6.8). The fact that (6.8) refines (6.7) is immediate and the proof is finished.

We have the following result as well. 
Theorem 6.6 Let $x, a, \alpha, \beta-\alpha \in(0, \infty)^{n}$. For any $u=:\left(u_{1}, \ldots, u_{n}\right) \in(0, \infty)^{n}$, we have

$$
\begin{aligned}
& \int_{(0, \infty)^{n}} \prod_{i=1}^{n} p_{i}^{u_{i}-1} B_{p}^{(\alpha, \beta)}(x ; a) d p \\
& \geq B(x, a) \Gamma(u)\left(\prod_{i=1}^{n} a_{i}^{u_{i}}\right) \prod_{i=1}^{n}{ }_{1} F_{1}\left(\beta_{i}-\alpha_{i} ; \beta_{i} ; a_{i}\right)
\end{aligned}
$$

where we set $d p=: d p_{1} \cdots d p_{n}$ and $\boldsymbol{\Gamma}(u)$ is defined by (2.2). In particular, if $u=e=:(1, \ldots, 1)$ then one has

$$
\int_{(0, \infty)^{n}} B_{p}^{(\alpha, \beta)}(x ; a) d p \geq B(x, a)\left(\prod_{i=1}^{n} a_{i}\right) \prod_{i=1}^{n}{ }_{1} F_{1}\left(\beta_{i}-\alpha_{i} ; \beta_{i} ; a_{i}\right) .
$$

Proof Multiplying (6.7) by $\prod_{i=1}^{n} p_{i}^{u_{i}-1}$, we obtain

$$
\prod_{i=1}^{n} p_{i}^{u_{i}-1} B_{p}^{(\alpha, \beta)}(x ; a) \geq B(x, a) \prod_{i=1}^{n} p_{i}^{u_{i}-1} e^{-p_{i} / a_{i}} \prod_{i=1}^{n}{ }_{1} F_{1}\left(\beta_{i}-\alpha_{i} ; \beta_{i} ; a_{i}\right)
$$

By integrating (6.12) with respect to $p \in(0, \infty)^{n}$, we get

$$
\begin{aligned}
& \int_{(0, \infty)^{n}} \prod_{i=1}^{n} p_{i}^{u_{i}-1} B_{p}^{(\alpha, \beta)}(x ; a) d p \\
& \quad \geq B(x, a) \prod_{i=1}^{n}{ }_{1} F_{1}\left(\beta_{i}-\alpha_{i} ; \beta_{i} ; a_{i}\right) \int_{(0, \infty)^{n}} \prod_{i=1}^{n} p_{i}^{u_{i}-1} e^{-p_{i} / a_{i}} .
\end{aligned}
$$

Making the change of variables $p_{i}=a_{i} v_{i}, i=1, \ldots, n$ in the right-hand side integral of (6.13), we obtain

$$
\begin{aligned}
& \int_{(0, \infty)^{n}} \prod_{i=1}^{n} p_{i}^{u_{i}-1} B_{p}^{(\alpha, \beta)}(x ; a) d p \\
& \geq B(x, a) \prod_{i=1}^{n}{ }_{1} F_{1}\left(\beta_{i}-\alpha_{i} ; \beta_{i} ; a_{i}\right) \prod_{i=1}^{n} a_{i}^{u_{i}} \int_{(0, \infty)^{n}} \prod_{i=1}^{n} v_{i}^{u_{i}-1} e^{-v_{i}} d \nu .
\end{aligned}
$$

Hence (6.10). Taking $u_{i}=1 ; i=1, \ldots, n$ in (6.10), we get (6.11). The proof is complete.

The following result may be recited as well.

Theorem 6.7 Let $x, a, \alpha, \beta-\alpha \in(0, \infty)^{n}$, and $p \in[0, \infty)^{n}$. Then we have

$$
\frac{B_{p}^{(\alpha, \beta)}(x ; a)}{B(x ; a)} \geq \prod_{i=1}^{n}{ }_{1} F_{1}\left(\alpha_{i} ; \beta_{i} ;-\frac{p_{i}}{a_{i}}\right)
$$


Proof By (1.8), we have

$$
\begin{aligned}
{ }_{1} F_{1}\left(\alpha_{i} ; \beta_{i} ;-\frac{a_{i}}{t_{i}}-p_{i} \frac{t_{i}}{a_{i}}\right) \\
\quad=\frac{\Gamma\left(\beta_{i}\right)}{\Gamma\left(\alpha_{i}\right) \Gamma\left(\beta_{i}-\alpha_{i}\right)} \int_{0}^{1} u^{\alpha_{i}-1}(1-u)^{\beta_{i}-\alpha_{i}-1} e^{-\frac{a_{i}}{t_{i}} u} e^{-p_{i} \frac{t_{i}}{a_{i}} u} d u .
\end{aligned}
$$

Since $0<t_{i} \leq 1$ for any $i=1, \ldots, n$ and $u \in[0,1]$, one has

$$
e^{-\frac{a_{i}}{t_{i}} u} \geq e^{-\frac{a_{i}}{t_{i}}} \quad \text { and } \quad e^{-p_{i} \frac{t_{i}}{a_{i}} u} \geq e^{-\frac{p_{i}}{a_{i}} u}
$$

Substituting these into (6.15) and then into (5.1), we get

$$
\begin{aligned}
B_{p}^{(\alpha, \beta)}(x ; a) \geq & \int_{E_{n-1}} \prod_{i=1}^{n} t_{i}^{x_{i}-1} e^{-\frac{a_{i}}{t_{i}}} d t \\
& \times \prod_{i=1}^{n} \frac{\Gamma\left(\beta_{i}\right)}{\Gamma\left(\alpha_{i}\right) \Gamma\left(\beta_{i}-\alpha_{i}\right)} \int_{0}^{1} u^{\alpha_{i}-1}(1-u)^{\beta_{i}-\alpha_{i}-1} e^{-\frac{p_{i}}{a_{i}} u} d u .
\end{aligned}
$$

This, with (1.15) and (1.8) again, yields (6.14). This completes the proof.

We end this section by stating the following result.

Theorem 6.8 Let $x, a, \alpha, \beta-\alpha \in(0, \infty)^{n}$. For any $u=:\left(u_{1}, \ldots, u_{n}\right) \in(0, \infty)^{n}$, we have

$$
\int_{(0, \infty)^{n}} \prod_{i=1}^{n} p_{i}^{u_{i}-1} B_{p}^{(\alpha, \beta)}(x ; a) d p \geq B(x, a) \Gamma^{(\alpha, \beta)}(u) \prod_{i=1}^{n} a_{i}^{u_{i}}
$$

where $\Gamma^{(\alpha, \beta)}(u)$ is defined by (2.4). In particular, taking $u=e=:(1,1, \ldots, 1)$, we get

$$
\int_{(0, \infty)^{n}} B_{p}^{(\alpha, \beta)}(x, a) d p \geq B(x, a) \Gamma^{(\alpha, \beta)}(e) \prod_{i=1}^{n} a_{i} .
$$

Proof It is similar to that of Theorem 6.6, by utilizing (6.14). We therefore leave the details for the reader.

Acknowledgements

The authors would like to thank the three anonymous referees for their valuable comments and suggestions which have substantially improved the final version of this manuscript.

Funding

Not applicable.

Availability of data and materials

Not applicable.

Competing interests

The authors declare that they have no competing interests. 


\section{Author details}

${ }^{1}$ Department of Mathematics, Science Faculty, Qassim University, Qassim, Saudi Arabia. ${ }^{2}$ Department of Mathematics, Science Faculty, Moulay Ismail University, Meknes, Morocco. ${ }^{3}$ Department of Mathematics, Science Faculty, Taibah University, Medinah, Saudi Arabia.

\section{Publisher's Note}

Springer Nature remains neutral with regard to jurisdictional claims in published maps and institutional affiliations.

Received: 23 October 2020 Accepted: 10 May 2021 Published online: 20 May 2021

\section{References}

1. Alzer, H.: Inequalities for the beta function in $n$ variables. ANZIAM J. 44, 609-923 (2003)

2. Anderson, G.D., Vamanmurthy, M.K., Vuorinen, M.K.: Conformal Invariants, Inequalities, and Quasiconformal Maps. Wiley, New York (1997)

3. Andrews, G.E., Askey, R., Roy, R.: Special Functions. Cambridge University Press, Cambridge (1999)

4. Andrews, L.C.: Special Functions for Engineers and Applied Mathematicians. MacMillan Co., New York (1985)

5. Bell, W.W.: Special Functions for Scientist and Engineers. Oxford Univ. Press, London (1968)

6. Carlson, B.C.: Special Functions of Applied Mathematics. Academic Press, New York (1977)

7. Chaudhry, M.A., Qadir, A., Rafique, M., Zubair, S.M.: Extension of Euler's beta function. J. Comput. Appl. Math. 78(1), 19-32 (1997)

8. Choi, J., Rathie, A.K., Parmar, R.K.: Extension of extended beta, hypergeometric and confluent hypergeometric functions. Honam Math. J. 36(2), 357-385 (2014)

9. Dragomir, S.S., Agarwal, R.P., Barnett, N.S.: Inequalities for beta and gamma functions via some classical and new integral inequalities. J. Inequal. Appl. 5, 103-165 (2000)

10. Grenié, L., Molteni, G.: Inequalities for the beta function. Math. Inequal. Appl. 18(4), 1427-1442 (2015)

11. Ivády, P.: On a beta function inequality. J. Math. Inequal. 6(3), 333-341 (2012)

12. Khan, N., Usman, T., Aman, M.: Extended beta, hypergeometric and confluent hypergeometric functions. Trans. Natl. Acad. Sci. Azerb. Ser. Phys.-Tech. Math. Sci. Math. 39(1), 83-97 (2019)

13. Khan, N., Usman, T., Aman, M.: Generalized Wright function and its properties using extended beta function. Tamkang J. Math. 51(4), 349-363 (2020)

14. Khan, N., Usman, T., Aman, M., Al-Omari, S., Araci, S.: Computation of certain integral formulas involving generalized Wright function. Adv. Differ. Equ. 2020, 491 (2020)

15. Khan, N.U., Usman, T., Aman, M.: Some properties concerning the analysis of generalized Wright function. J. Comput. Appl. Math. 376, $112840(2020)$

16. Lebedev, N.N.: Special Functions and Their Applications. Prentice Hall, New York (1965)

17. Luke, Y.L: The Special Functions and Their Approximations. Academic Press, New York (1969)

18. Macdonald, A.D.: Proprieties of the confluent hypergeometric function. Technical Report 84 , Research Laboratories of Electronics, 1948

19. Özergin, E., Özarslan, M.A., Altin, A.: Extensions of gamma, beta and hypergeometric functions. J. Comput. Appl. Math 235(16), 4601-4610 (2011)

20. Rainville, E.: Special Functions. Chelsea, New York (1971)

21. Raïssouli, M., El-Soubhy, S.I. Extending and refining some inequalities for the beta function in $n$ variables. Submitted

22. Raïssouli, M.. El-Soubhy, S.I: Extension of the beta function in $n$ variables, applications for orthogonal polynomials. Submitted

23. Whittaker, E.T., Watson, G.N.: A Course of Modern Analysis, 4th edn. Cambridge University Press, New York (1962)

\section{Submit your manuscript to a SpringerOpen ${ }^{\circ}$ journal and benefit from:}

- Convenient online submission

- Rigorous peer review

- Open access: articles freely available online

- High visibility within the field

Retaining the copyright to your article

Submit your next manuscript at $\boldsymbol{~ s p r i n g e r o p e n . c o m ~}$ 